\title{
The Potential of a Multi-fidelity Approach to Gas Turbine Combustor Design Optimization
}

\author{
David J.J. Toal, Xu Zhang \& \\ Andy J. Keane \\ Faculty of Engineering \& Physical Sciences \\ University of Southampton \\ Southampton, U.K. \\ SO16 7QF \\ Chin Yik Lee \& Marco Zedda \\ Rolls-Royce Plc. \\ Derby, U.K. \\ DE24 8BJ
}

\begin{abstract}
The desire to reduce gas turbine emissions drives the use of design optimization approaches within the combustor design process. However, the relative cost of combustion simulations can prohibit such optimizations from being carried out within an industrial setting. Strategies which can significantly reduce the cost of such studies can enable designers to further improve emissions performance.

The following paper investigates the application of a multi-fidelity surrogate modelling approach to the design optimization of a typical gas turbine combustor from a civil airliner engine. Results over three different case studies of varying problem dimensionality indicate that a multi-fidelity surrogate modelling based design optimization, whereby the simulation fidelity is varied by adjusting the coarseness of the mesh, can indeed improve optimization performance. These results indicate that such an approach has the potential to significantly reduce design optimization cost whilst achieving similar, or in some cases superior, design performance.
\end{abstract}

\footnotetext{
Nomenclature

1 Vector of ones of length $n$

C Multi-fidelity Kriging correlation matrix

$d$ Number of dimensions

d Difference vector

$n \quad$ Number of sample points

$p$ Kriging smoothness parameter

$\boldsymbol{R}$ Correlation matrix

$\boldsymbol{r}$ Correlation vector

$\boldsymbol{x}$ Vector of design variables

$\boldsymbol{Y}$ Combined vector of cheap and expensive data

$y$ Objective function value

$Z$ Gaussian process

$\lambda$ Loss coefficient

$\theta \quad$ Kriging rate of correlation decrease

$\phi$ Concentrated log-likelihood

$\hat{\mu} \quad$ Kriging mean

$\hat{\sigma}^{2} \quad$ Kriging variance
}

*Corresponding author: djjt@ soton.ac.uk 
$c$ Cheap or low-fidelity function evaluation

$d$ Difference between low and high fidelity

$e$ Expensive or high-fidelity function evaluation

\section{Introduction}

The application of optimization techniques to combustor design has the potential to unlock improvements in combustor performance, reduce emissions, increase turbine life and reduce fuel consumption. However, the cost of the computational simulations required as part of such an optimization can be prohibitively expensive thereby reducing the scope of any design study and hence the potential level of design improvement. Design optimization approaches which can reduce the cost of such studies are, therefore, very attractive.

Surrogate modelling approaches [1], whereby a cheap analytical model which predicts how the objective function or constraints vary with changing design variables are very useful at reducing the overall cost of such design studies. Whilst such techniques have been applied to combustor design studies throughout the literature the application of multi-fidelity surrogate modelling approaches to combustor design remain rare. Such an approach enables multiple levels of simulation fidelity to be fused together to enhance the accuracy of a surrogate model. If the fidelity levels are sufficiently well correlated [2] this can both accelerate the convergence of an optimization and reduce the total simulation cost. Whilst multi-fidelity surrogate model based optimizations of combustors have been demonstrated within the literature in the past, the current paper represents the first time this has been performed for the optimization of emissions on a geometry of a fidelity similar to that employed during a typical industrial design process.

Formal design optimization approaches have been applied to the design of gas turbine combustion systems throughout the literature with the fidelity of the simulations employed in such studies varying considerably from simple aerothermal network models, to 2D axisymmetric and 3D CFD simulations. Of course, the choice and fidelity of simulation will depend, very much, on the design stage at which the optimization is performed.

Aerothermal network based design optimizations, given their relative inexpense, tend to be performed at the conceptual design stage. Rogero and Rubini [3], for example, employed an aerothermal network model and an evolutionary optimization algorithm to optimize for 22 performance targets while minimizing cooling flow and NOx emissions. Wyse et al. [4] employed a similar network model of a combustion system to minimise NOx and CO through a Tabu search while, more recently, Saboohi et al. [5] employed a chemical network model within a multi-objective optimization attempting to also reduce NOx and CO.

Other researchers have employed 2D axisymmetric CFD simulations within multi-objective combustor optimizations. As these simulations represent a considerable cost increase over an aerothermal network model, direct optimization using the objective function becomes infeasible and surrogate modelling strategies are employed to reduce the optimization cost. Torkzadeh et al. [6], for example, employed a surrogate modelling approach in combination with NSGA-II [7] to optimize a combustor for efficiency, NOx and CO. Recently Amani et al. [8] employed a similar surrogate modelling base strategy in a four variable optimization of a combustor for minimum NOx production.

Moving up the fidelity scale, other researchers have employed 3D RANS CFD within combustor design optimization frameworks. The work of Duchaine et al. [9] is perhaps one of the earliest examples of this with a, relatively small, by today's standard, 3D CFD simulation of $1.1 \mathrm{~m}$ elements, being employed within a Kriging based surrogate model optimization for both combustor efficiency and exit temperature profile. Motsamai et al. [10] performed a similar five variable optimization of the exit temperature profile of a combustor using 3D RANS simulations. Laranci et al. [11] also employed 3D RANS simulations in their optimization of exit temperature and NOx but within a simpler two variable, full factorial, DoE approach instead of a formal optimization. Recently Briones et al. [12] and Thomas et al. [13] both optimized combustors considering the exit temperature profile and pressure losses using 3D RANS simulations, with 9 and 15 geometry parameters respectively, but did not consider emissions reduction.

As noted above, multi-fidelity design optimization approaches have been applied to combustor design in the past. The work of Wankhede et al. [14] represents the first application of such an approach, however, this employed only two design variables and optimized the exit temperature profile of a relatively simple 2D flame stabilization step. Despite the apparent simplicity of the geometry, their work successfully demonstrated that data from steady and unsteady turbulent combustion simulations could be fused together effectively. Employing the Prometheus combustor design system [15], Zhang et al. [16] demonstrated that multi-fidelity optimization of a combustor could be performed using isothermal simulations to minimize pressure losses. In this instance the fidelity of the simulation was varied by switching between a single and double sector model of the combustor. Toal et al. [17] extended this work to include reactions within the CFD simulation. In this case their optimization considered both the combustor exit temperature profile and pressure loss. While 3D RANS simulations were used to calculate both the temperature profile and the pressure losses an aerothermal network prediction of the pressure loss provided a low fidelity data set which accelerated the design optimization. Both the work of Zhang et al. [16] and Toal et 
Copyright (ai 207$]$ employed combustor geometry of a significantly greater fidelity than is typically found within the literature with the inclusion of the prediffuser and the swirler vanes and passages.

The current article represents an improvement on the state-of-the-art through a combination of three areas. Firstly, the fidelity of the geometry is considerably greater than the majority of the combustor design optimization examples found within the literature through the inclusion of both the combustor and the outer and inner annuli. Feed hole flows are therefore modelled explicitly and not defined as boundary conditions. Secondly, both NOx and soot are subjects of the presented design optimizations and to date this has not previously been demonstrated either via a single or multi-fidelity optimization. Finally, the multi-fidelity approach, whereby, the fidelity of the 3D CFD mesh is varied has not previously been demonstrated for a combustor simulation.

The following paper commences by providing a brief overview of the mathematics behind both the single and multifidelity surrogate modelling strategies employed within the paper. The combustor case study is then described in detail both in terms of the parametric geometry and the simulation set-up. The differences between both simulation fidelities are also highlighted at this point. The results for three different optimizations of increasing complexity (in terms of the number of design variables) employing this combustor are then presented. In each case results for two single fidelity optimization strategies of varying simulation cost are presented along with the results for a multi-fidelity optimization. General conclusions from the studies are then presented.

\section{Multi-fidelity Surrogate Modelling}

Given the expense of the combustion CFD simulations involved, both the single and multi-fidelity design optimization approaches presented within this paper follow a surrogate modelling philosophy. That is to say, a global optimization algorithm is applied, not directly to the objective function calculation, but instead to a surrogate model of the objective function. This surrogate is an analytical model which attempts to predict the variation in the objective function as the design parameters are varied. The process commences with the definition of a sampling plan via some form of design of experiments approach. The true objective function is determined at each of these points and used to construct an initial surrogate model. The model is optimized based on some metric via a global optimization approach with promising designs then evaluated using the true objective function. This data is then used to 'update' the surrogate model and the search performed again on this corrected model. Constrained optimizations can be performed in exactly the same manner but with additional surrogate models of each constraint being constructed.

In the context of the present study, CFD simulations of a set of combustor designs defined by a design of experiments are performed the data from which is then used to construct surrogate models of NOx, soot and pressure loss. These surrogates are then searched using a constrained global optimization for potential promising designs which are then simulated using CFD. The results from these simulations are then used to update and correct the surrogate prediction in regions of interest.

Both the single and multi-fidelity surrogate modelling approaches employed here are based upon variants of the popular method known as Kriging [18]. Within such a model the assumption is that sample points close together within the design space are more highly correlated than those further apart. This correlation is modelled through a correlation matrix $\boldsymbol{R}$ with entries given by,

$$
\boldsymbol{R}_{i j}=\exp \left(-\sum_{l=1}^{d} 10^{\boldsymbol{\theta}^{(l)}}\left\|\boldsymbol{x}_{i}^{(l)}-\boldsymbol{x}_{j}^{(l)}\right\|^{\boldsymbol{p}^{(l)}}\right),
$$

where $\theta$ describes the rate of correlation decrease and $p$ describes a smoothness parameter. An additional parameter $\lambda$ is often appended to the diagonal of this matrix to enable the resulting model to regress [19], something which is very important when constructing a model from simulations containing noise. All of the models constructed within the current paper include such a regressing term.

Including the regression term there are a total of $2 d+1$ hyperparameters which must be determined, where $d$ represents the number of input variables. Within the following paper a maximum likelihood approach is taken to determine these parameters with an optimization of the likelihood function,

$$
\phi=-\frac{n}{2} \ln \left(\hat{\boldsymbol{\sigma}}^{2}\right)-\frac{1}{2} \ln (|\boldsymbol{R}|),
$$

where the variance is defined by,

$$
\hat{\sigma}^{2}=\frac{1}{n}(\boldsymbol{y}-\mathbf{1} \hat{\mu})^{T} \boldsymbol{R}^{-1}(\boldsymbol{y}-\mathbf{1} \hat{\mu}),
$$




$$
\hat{\mu}=\frac{\mathbf{1}^{T} \boldsymbol{R}^{-1} \boldsymbol{y}}{\mathbf{1}^{T} \boldsymbol{R}^{-1} \mathbf{1}}
$$

being performed by a hybridized particle swarm algorithm [20] which exploits an adjoint of the likelihood function to accelerate convergence [21].

Having determined an appropriate set of hyperparameters the model can be used to predict either the objective function or constraint value at an unknown point via,

$$
y\left(\boldsymbol{x}^{*}\right)=\hat{\mu}+\boldsymbol{r}^{T} \boldsymbol{R}^{-1}(\boldsymbol{y}-\mathbf{1} \hat{\mu}),
$$

where $\boldsymbol{r}$ represents a vector of correlations between the known sample points and an unknown point $\boldsymbol{x}^{*}$. Although the current paper employs only the Kriging predictor it is worth pointing out that the Kriging model's error prediction enables other metrics to be derived including, for example, expected improvement and probability of improvement. The interested reader can find more information on these approaches in the work of Jones [18].

In order to include data from differing levels of simulation fidelity the approach outlined by Kennedy and O'Hagan [22], referred to here as co-Kriging, is adopted. Here the assumption is that a Gaussian process of only the low fidelity data can be scaled by $\rho$ and appended to a second Gaussian process of the difference between the low and high fidelity data. This combination,

$$
Z_{e}(\boldsymbol{x})=\rho Z_{c}(\boldsymbol{x})+Z_{d}(\boldsymbol{x})
$$

results in a Gaussian multi-fidelity prediction of the high fidelity data.

The construction of such a model commences with the construction of a single fidelity Kriging model of the low fidelity data using the method outlined above. A second Kriging model is then constructed of the differences between the low and high fidelity data with these differences given by,

$$
\boldsymbol{d}=\boldsymbol{y}_{e}-\rho \boldsymbol{y}_{c}\left(\boldsymbol{X}_{e}\right)
$$

Once again a maximum likelihood approach is taken to determine the hyperparameters of this second model but unlike the low fidelity model the additional scaling parameter, $\rho$, must also be determined. As with the single fidelity Kriging model the hyperparameters for both the low fidelity and difference models are optimized using a hybrid particle swarm. A modified version of the adjoint of the likelihood function is employed to calculate the derivative of the scaling parameter $\rho$, further details of which can be found in Toal et al. [23].

With the two sets of hyperparameters determined a prediction of the high fidelity response at an unknown point can be calculated via,

$$
y_{e}\left(\boldsymbol{x}^{*}\right)=\hat{\mu}+\boldsymbol{c}^{T} \boldsymbol{C}^{-1}(\boldsymbol{y}-1 \hat{\mu})
$$

where the correlation matrix $\boldsymbol{C}$ is defined as,

$$
\boldsymbol{C}=\left(\begin{array}{cc}
\sigma_{c}^{2} \boldsymbol{R}_{c}\left(\boldsymbol{X}_{c}, \boldsymbol{X}_{c}\right) & \rho \sigma_{c}^{2} \boldsymbol{R}_{c}\left(\boldsymbol{X}_{c}, \boldsymbol{X}_{e}\right) \\
\rho \sigma_{c}^{2} \boldsymbol{R}_{c}\left(\boldsymbol{X}_{e}, \boldsymbol{X}_{c}\right) & \rho^{2} \sigma_{c}^{2} \boldsymbol{R}_{c}\left(\boldsymbol{X}_{e}, \boldsymbol{X}_{e}\right)+\sigma_{d}^{2} \boldsymbol{R}_{d}\left(\boldsymbol{X}_{e}, \boldsymbol{X}_{e}\right)
\end{array}\right),
$$

the mean is given by,

$$
\hat{\mu}=\frac{\mathbf{1}^{T} C^{-1} \boldsymbol{Y}}{\mathbf{1}^{T} C^{-1} \mathbf{1}},
$$




$$
\boldsymbol{c}=\left[\begin{array}{c}
\rho \hat{\sigma}_{c}^{2} \boldsymbol{R}_{c}\left(\boldsymbol{X}_{c}, \boldsymbol{x}^{*}\right) \\
\rho^{2} \hat{\boldsymbol{\sigma}}_{c}^{2} \boldsymbol{R}_{c}\left(\boldsymbol{X}_{e}, \boldsymbol{x}^{*}\right)+\hat{\boldsymbol{\sigma}}_{d}^{2} \boldsymbol{R}_{d}\left(\boldsymbol{X}_{e}, \boldsymbol{x}^{*}\right)
\end{array}\right] .
$$

As per the single fidelity model the multi-fidelity Kriging model also offers a useful prediction of the error in the model from which other updating metrics, such as expected improvement can be calculated but in the following optimizations only the predictor is employed. In the following optimizations all updates are located by searching either the single or multi-fidelity surrogate model using a genetic algorithm, in this case a version of NSGA-II [7].

All of the surrogate modelling and optimization algorithms employed within the current paper are embedded within the proprietary Rolls-Royce optimization package OPTIMATv3.

\section{Combustor Case Study}

The gas turbine combustor geometry considered as part of the current study is representative of that found within a typical modern civil airliner engine. Illustrated in Figure 1, the geometry includes the combustor along with the inner and outer annuli. Geometry upstream of the meterpanel, such as the prediffuser, fuel spray nozzle and swirler passages has been excluded from the model.

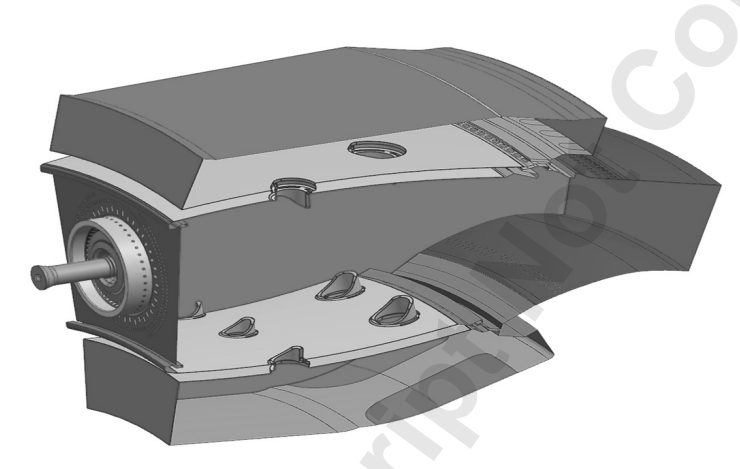

Fig. 1. An illustration of an example combustor CFD fluid volume.

A parametric version of this combustor geometry has been implemented within Siemens NX and is capable of adjusting the individual positions and dimensions of each of the ports present on the walls of the combustor.

In all cases an unstructured tetrahedral mesh with hexahedral core conversion is constructed using the commercial meshing package Ansys ICEM CFD. In the present study the size of the CFD mesh is varied in order to vary the fidelity of the simulation. The baseline high fidelity CFD mesh consists of approximately $16.7 \mathrm{M}$ elements whereas the low fidelity CFD mesh consists of approximately $3.6 \mathrm{M}$ elements. The $16.7 \mathrm{M}$ element meshing strategy was developed as a result of a standard mesh convergence study while the $3.6 \mathrm{M}$ element meshing strategy was the result of a study balancing cost reduction over the $16.7 \mathrm{M}$ element mesh and correlation between the resulting design spaces for the two variable case study detailed in Section 5. As outlined by Toal [2] the choice of low fidelity model is important to the success of a multifidelity optimization, with poorly correlated models potentially misleading the optimization and fidelities of a similar expense negating any improvement in optimization cost.

Each combustor design is simulated using the proprietary Rolls-Royce CFD package PRECISE-UNS [24]. The K- $\varepsilon$ realizable turbulence model is used with simulations running for a total of 5000 iterations where fuel is introduced into the model after 500 iterations and ignition occurring after 600. Combustion is modelled via the flamelet-generated manifold technique. A set of inlet velocity profiles are used to define the flow at the inlets to both annuli and at the exit of each of the swirler passages. Each of these profiles remains constant as the geometry is varied. All simulations are performed at a condition representative of $100 \%$ throttle for a large civil airliner gas turbine engine.

All simulations are carried out using 16 compute cores on the University of Southampton Spitfire cluster with up to 50 simulations being performed in parallel. Given the considerable reduction in element count between the two fidelities a single low fidelity simulation can be completed in approximately $21.6 \%$ of the time taken to perform a high fidelity simulation. This is inclusive of the time take to generate the mesh.

Post-processing routines within PRECISE-UNS are used to calculate emissions indices for NOx and soot while Paraview is used to calculate the inner and outer wall losses. 


\section{Copyright (f) 2020 ase Study Overview}

Given the expense of each CFD simulation it becomes difficult to perform enough optimizations to gain an appreciation of the average performance of each strategy. There is always, a possibility that a member of the initial sampling plan locates the global optimum or a design very close to the global optimum which can skew any conclusions; the so-called 'getting lucky' scenario. In the current paper the performance of each optimization strategy is assessed in two ways.

The first two case studies do not employ actual CFD simulations as part of the optimization. Instead a globally accurate surrogate model is constructed for the objective function and all constraints from a relatively large initial sampling plan. The number of samples lying between 40 and 80 times the number of design variables. This model is then called instead of a CFD simulation as part of the design optimizations thereby enabling many such optimizations to be carried out within a reasonable time frame and statistics to be taken over multiple initial sampling plans. However, it should be observed that as no CFD simulation of the final design is actually carried out the quoted improvements in performance may not actually be realizable and depend entirely on the accuracy of the original surrogate model.

The third and final case study however, is performed as it would be in reality with CFD simulations being used directly within the initial sampling plan and subsequent updates to the surrogates. While statistics cannot be calculated using this case it introduces a considerable level of realism with both optimization strategies now having to cope with noise in the responses and failed simulations.

Failed simulations in this particular optimization problem tend to stem from either a failure of the meshing process resulting in poor quality elements and therefore a divergence of the CFD simulation, or from a lack of convergence of the CFD simulation. Typically, $10 \%$ of the simulations performed as part of the DoE will fail due to one of these errors. Whilst the results from failed simulations are not included in the construction of any of the surrogate models the design variables corresponding to these simulations are used as a constraint within the search stage to ensure that any updates are at least $1 \%$ away from a previously evaluated design. This prevents the optimization from entering into a loop where it continually attempts to evaluate a design which results in a meshing or simulation failure.

In all of the case studies the minimization of NOx is considered as the objective function with change in wall pressure losses constrained to be within $15 \%$ of that of the baseline combustor. There are two 'flavours' of this basic optimization, one where a constraint on soot production is included and one where it is ignored. This particular constraint limits the production of soot to be no greater than that of the baseline design. It's inclusion has the impact of severely limiting the level of NOx improvement possible as a reduction in NOx is often met with an increase in soot production.

\section{2D Case Study}

The first, and simplest, case study involves a two variable design optimization. In this case the inner and outer ports are grouped together and permitted to move axially along the length of their respective walls.

As already noted above both the single and multi-fidelity optimizations will be performed on an initial surrogate model constructed from a relatively large sampling plan. In this case 81 combustor designs are simulated throughout the two variable design space comprising of the baseline design and an 80 point optimal space filling sampling plan. An identical sampling plan is employed for both the low and high fidelity simulations with Kriging surrogate models of NOx, soot and wall pressure losses constructed for both fidelities.

Tables 1 and 2 below indicate the accuracy of the resulting surrogate models for each quantity for both fidelities. Surrogate accuracy, in this instance, is determined via a leave one out cross-validation from which Pearson's $r^{2}$ correlation, percentage root mean square error (RMSE) and percentage maximum absolute error (MAE) can be calculated. In all cases the correlation between the surrogate and CFD results are relatively high and the magnitudes of the errors relatively low with significant differences between CFD and prediction a result of noise in the CFD result.

Table 1. High fidelity 2D surrogate model accuracy

\begin{tabular}{llll}
\hline \hline Parameter & $\mathrm{r}^{2}$ & \%RMSE & \%MAE \\
\hline NOx & 0.941 & 5.52 & 21.6 \\
Soot & 0.880 & 8.09 & 32.0 \\
Inner $\Delta \mathrm{P}$ & 0.896 & 7.22 & 40.3 \\
Outer $\Delta \mathrm{P}$ & 0.963 & 5.63 & 34.8 \\
\hline
\end{tabular}

The work of Toal [2] demonstrated the importance of a relatively high level of correlation between different levels of simulation fidelity in order for a multi-fidelity approach to be effective. Low levels of correlation can, for example, 
Table 2. Low fidelity 2D surrogate model accuracy

\begin{tabular}{llll}
\hline \hline Parameter & $\mathrm{r}^{2}$ & \%RMSE & \%MAE \\
\hline NOx & 0.926 & 6.22 & 14.8 \\
Soot & 0.725 & 11.0 & 35.8 \\
Inner $\Delta \mathrm{P}$ & 0.918 & 5.94 & 28.1 \\
Outer $\Delta \mathrm{P}$ & 0.976 & 4.91 & 23.6 \\
\hline
\end{tabular}

result in the low fidelity data distorting the prediction of the high fidelity response and considerably hampering optimization performance. To that end Table 3 presents the level of correlation between the two fidelities in terms of both the Kriging models constructed using the 81 sample points and between the 81 sample points themselves.

Table 3. Correlations between low and high fidelity models across the two variable design space

\begin{tabular}{lll}
\hline \hline Parameter & $\mathrm{r}^{2}$ (surrogates) & $\mathrm{r}^{2}$ (simulations) \\
\hline NOx & 0.920 & 0.880 \\
Soot & 0.879 & 0.766 \\
Inner $\Delta \mathrm{P}$ & 0.962 & 0.940 \\
Outer $\Delta \mathrm{P}$ & 0.990 & 0.976 \\
\hline
\end{tabular}

Rather unsurprisingly, given the previous results of Toal et al. [17], the wall pressure losses exhibit a relatively high level of correlation between the two simulation fidelities. The level of correlation between simulation fidelities in terms of both the NOx and soot responses is also relatively high while the correlations between the surrogate models are very similar to those between the raw simulation data.



(a)



(b)

Fig. 2. High (a) and low (b) fidelity percentage variations in NOx, relative to the baseline design.

To further illustrate the similarity between the NOx and soot responses both the high and low fidelity surrogate models are presented below, where $x_{1}$ and $x_{2}$ denote the normalized variation in the position of the inner and outer ports respectively. Figure 2 compares the contours of percentage variation in NOx relative to the baseline design obtained from both the high and low fidelity models. While the responses are by no means identical one can clearly observe a considerable similarity between them. Figure 3 compares the contours of percentage variation in soot production relative to the baseline design 


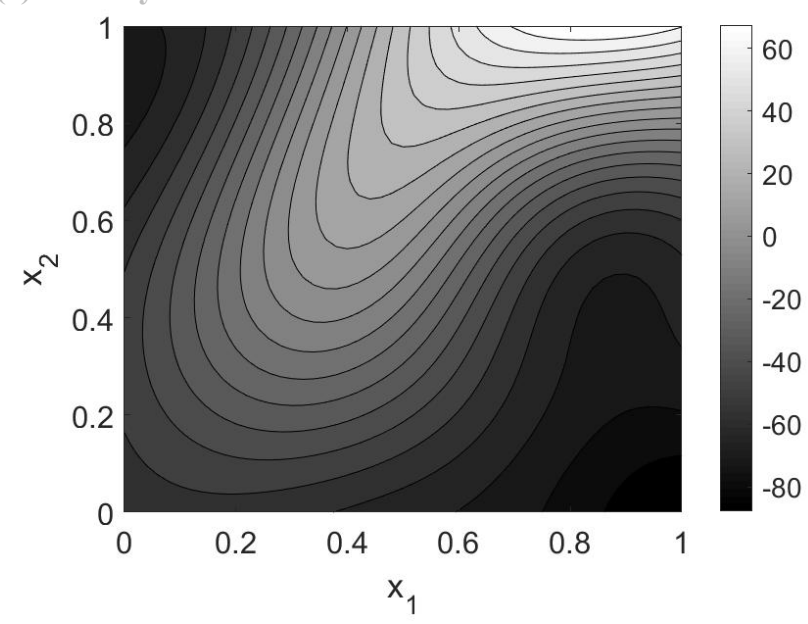

(a)

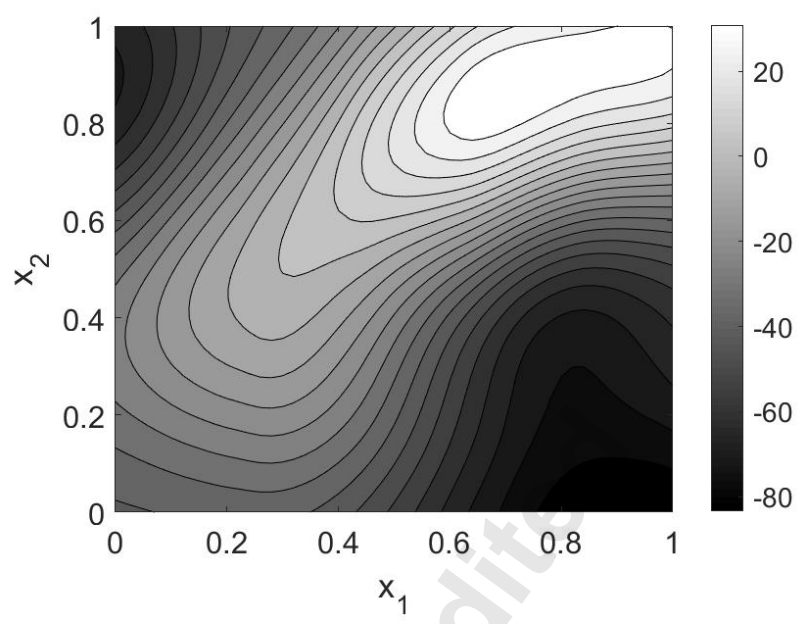

(b)

Fig. 3. High (a) and low (b) fidelity percentage variations in soot, relative to the baseline design.

obtained from both fidelities. Again, whilst there are clear differences in terms of the magnitude, the overall trend is very similar.

As described above, by employing an accurate set of surrogate models instead of CFD simulations to compare single and multi-fidelity approaches, a large number of such optimizations can be performed which enables a better understanding of the 'average' performance of each strategy. As part of this two variable case three different optimization strategies will therefore be performed 50 times each commencing from a different initial sampling plan.

The first strategy employs only high fidelity data commencing from a 20 point Latin hypercube sampling plan with 10 further updates to the surrogate model performed. The second strategy also employs only high fidelity data but commences from a smaller 10 point sampling plan which again is subject to 10 updates as the optimization progresses. The third strategy is a multi-fidelity approach commencing with a 10 point sampling plan from which only low fidelity data is employed. This sampling plan is identical to that used in the second strategy with an optimal, three point, space-filling sub-set of this sapling plan used to evaluate the high fidelity data. As with the other two strategies 10 update cycles are performed but each update point is evaluated both from the low and high fidelity model. Assuming the costs of each low and high fidelity evaluation are equivalent to that of actual CFD simulation means that the multi-fidelity Co-Kriging approach is approximately 57\% of the cost of the 20 point single fidelity approach.

Two different optimizations are performed using each of the three strategies. The first is a minimization of NOx production subject to a constraint on wall pressure losses, the second includes soot production as an additional constraint. The average percentage reduction in NOx production as a result of each optimization is presented in Table 4 with the mean optimization histories for each strategy presented in Figure 4.

Table 4. Two variable optimization strategy cost and average percentage of improvement in NOx over 50 different optimizations with and without soot constraint included

\begin{tabular}{llll}
\hline \hline Strategy & Cost & $\begin{array}{l}\text { Improvement } \\
\text { (w/o soot) }\end{array}$ & $\begin{array}{l}\text { Improvement } \\
\text { (with soot) }\end{array}$ \\
\hline 20pt Krig & $100 \%$ & $8.09 \%$ & $4.09 \%$ \\
10pt Krig & $67 \%$ & $8.09 \%$ & $4.03 \%$ \\
3+10pt Co-Krig & $57 \%$ & $8.08 \%$ & $4.09 \%$ \\
\hline
\end{tabular}

Comparing the results presented in Table 4 there is little obvious difference in the performance of the final designs. All three strategies achieve over an $8 \%$ reduction in NOx when the soot constraint is ignored and more than a $4 \%$ reduction when the soot constraint is included. Figures 2 and 3 clearly indicate that the improvement in NOx is limited when the soot constraint is included and this is reflected in the difference in the performance of the final designs. 


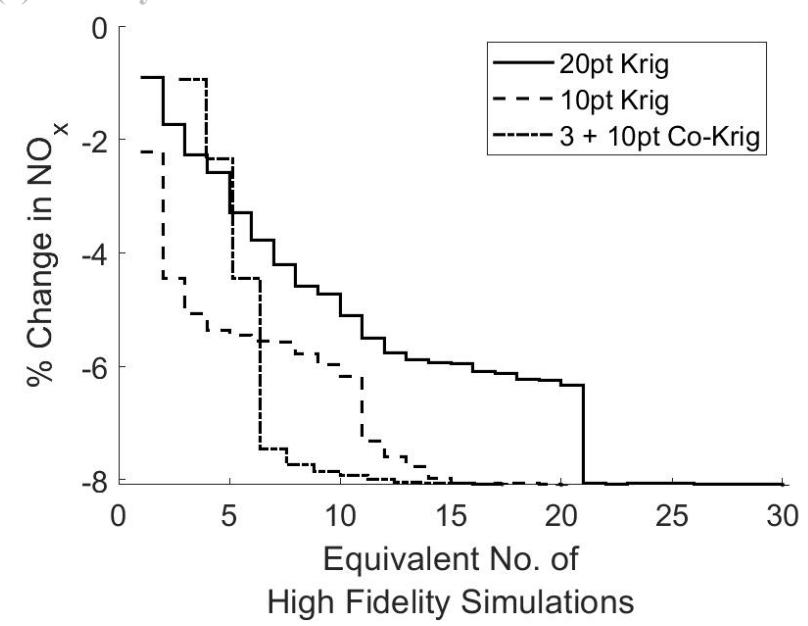

(a)

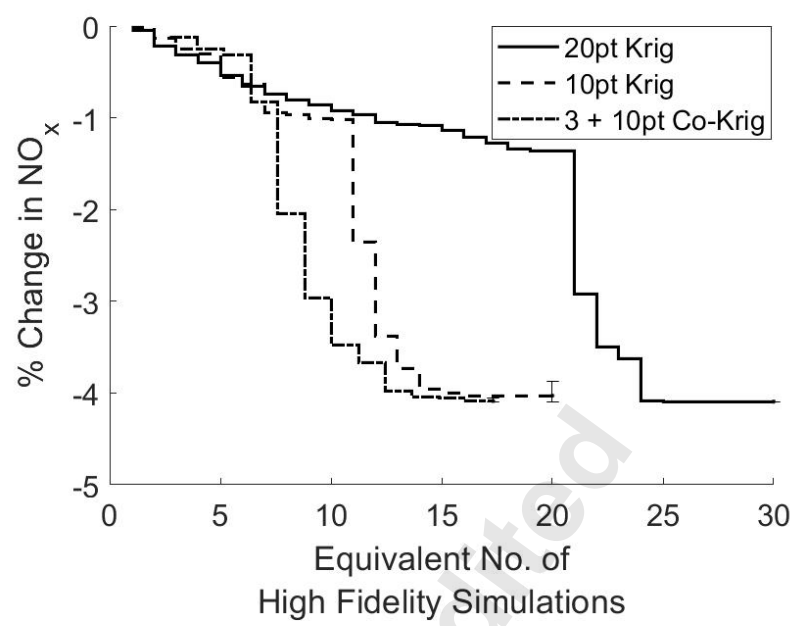

(b)

Fig. 4. Mean search histories for each 2D optimization strategy (a) without soot constraint and (b) with soot constraint active.

Of course, Table 4, only presents the final best design found not how efficiently it was obtained. The search histories in Figure 4 are presented as a function of the equivalent number of high fidelity simulations and include the cost of both the initial design of experiments sampling plan and any subsequent updates. For the single fidelity approaches this is a simple concept but this is complicated somewhat for the multi-fidelity optimization. Here each low fidelity evaluation is the equivalent of $21.6 \%$ the cost of a high fidelity evaluation, the search history for the multi-fidelity optimization is therefore shifted to the right by the equivalent of 7 low fidelity simulations to take into account the large low fidelity design of experiments. Each subsequent update is therefore treated as 1.216 high fidelity simulations as both low and high fidelity evaluations are made.

Included in all of the convergence history plots for both the two and eight variable case studies are the $95 \%$ bootstrap confidence bounds in the mean of the best design for the final iteration. In most cases, these bounds are very small relative to the overall percentage change in NOx.

Figure 4 demonstrates that regardless of the presence of the soot constraint the multi-fidelity approach convergences towards the optimum faster than either the 20 or 10 point single fidelity strategies. This is despite the fact that the multifidelity approach is considerably cheaper. Of the two optimization problems, the results indicate that convergence towards an optimum is improved more when the soot constraint is not present.

\section{8D Case Study}

Consider now an eight variable combustor optimization problem. In this case the ports are placed into four distinct groups based upon their proximity in the baseline geometry, see Figure 1. The two inner primary ports form a group, as do the two outer primary ports, the two inner secondary ports and the two outer secondary ports. The axial position and radii of each group of ports is controlled by a pair of design variables with the variables for each group independent of eachother. As per the previous two variable case study, surrogate models of NOx, soot and wall pressure losses will be constructed from a large sampling plan with multiple single and multi-fidelity design optimizations then evaluating these surrogates instead of carrying out an actual CFD simulation.

The underlying surrogate models are constructed from a large 640 point optimal Latin hypercube sampling plan the accuracies of which are presented in Tables 5 and 6. Once again, the accuracy of each model is calculated via a leave-oneout cross-validation. The results indicate that the high and low fidelity predictions of NOx and wall pressure losses correlate well with the simulations but there is a drop in accuracy for the surrogate model of soot production.

Table 7 illustrates the level of correlation between the low and high fidelity surrogate models and the 640 CFD simulation results used in their construction. As with the two variable case study the correlations between the different fidelity surrogates are similar to those from the CFD simulations in the majority of cases. The exception being soot production which indicates a much poorer level of correlation between the simulations that the surrogates. This coupled with the reduction in surrogate accuracy points to a considerable level of noise being present within the soot response which the surrogate models are struggling to represent. This reduction in accuracy raises some questions about the validity of any results employing the soot constraint but as before optimizations both with and without this constraint are considered.

Once again three optimization strategies are considered, two single fidelity and one multi-fidelity. The two single fidelity approaches employ, respectively, 80 and 40 point sampling plans with both then subject to a total of 40 update cycles. The 
Table 5. High fidelity 8D surrogate model accuracy

\begin{tabular}{llll}
\hline \hline Parameter & $\mathrm{r}^{2}$ & \%RMSE & \%MAE \\
\hline NOx & 0.805 & 7.33 & 51.4 \\
Soot & 0.632 & 10.5 & 78.4 \\
Inner $\Delta \mathrm{P}$ & 0.894 & 7.74 & 80.5 \\
Outer $\Delta \mathrm{P}$ & 0.946 & 5.52 & 76.7 \\
\hline
\end{tabular}

Table 6. Low fidelity 8D surrogate model accuracy

\begin{tabular}{llll}
\hline \hline Parameter & $\mathrm{r}^{2}$ & \%RMSE & $\% \mathrm{MAE}$ \\
\hline NOx & 0.803 & 7.00 & 31.4 \\
Soot & 0.522 & 11.5 & 87.1 \\
Inner $\Delta \mathrm{P}$ & 0.980 & 3.29 & 62.3 \\
Outer $\Delta \mathrm{P}$ & 0.968 & 4.14 & 82.3 \\
\hline
\end{tabular}

Table 7. Correlations between low and high fidelity models across the eight variable design space

\begin{tabular}{lll}
\hline \hline Parameter & $\mathrm{r}^{2}$ (surrogates) & $\mathrm{r}^{2}$ (simulations) \\
\hline NOx & 0.892 & 0.840 \\
Soot & 0.778 & 0.513 \\
Inner $\Delta \mathrm{P}$ & 0.994 & 0.932 \\
Outer $\Delta \mathrm{P}$ & 0.995 & 0.984 \\
\hline
\end{tabular}

multi-fidelity approach employs a 40 point sampling plan of the low fidelity data and a 10 point sampling plan of the high fidelity data. The 40 point sampling plan is an optimal space-filling subset of the 80 point plan and the 10 point sampling plan is an optimal space-filling subset of the 40 point plan. Relative costs of both strategies are presented in Table 8 along with the percentage reduction in NOx obtained on both optimization problems for each of the three strategies.

Table 8. Eight variable optimization strategy cost and average percentage of improvement in NOx over 50 different optimizations with and without soot constraint included

\begin{tabular}{llll}
\hline \hline Strategy & Cost & $\begin{array}{l}\text { Improvement } \\
\text { (w/o soot) }\end{array}$ & $\begin{array}{l}\text { Improvement } \\
\text { (with soot) }\end{array}$ \\
\hline 80pt Krig & $100 \%$ & $22.8 \%$ & $9.9 \%$ \\
40pt Krig & $67 \%$ & $22.7 \%$ & $8.7 \%$ \\
10+40pt Co-Krig & $56 \%$ & $22.6 \%$ & $7.9 \%$ \\
\hline
\end{tabular}

When the soot constraint is ignored all three strategies achieve a very similar level of reduction in NOx production of 22.6-22.8\%. However, when one observes the convergence histories for this particular problem presented in Figure 5(a) the multi-fidelity approach clearly converges more rapidly towards this optimum than either of the single fidelity approaches.

When the soot constraint is included there is a more pronounced difference in final level of NOx reduction achieved across the three strategies. The more expensive 80 point single fidelity strategy achieves a further $2 \%$ reduction upon that obtained with the multi-fidelity approach and a $1.2 \%$ reduction over the 40 point single fidelity approach. While the final reduction in NOx is not as great, Figure 5(b) indicates that the multi-fidelity approach converges more rapidly than either 


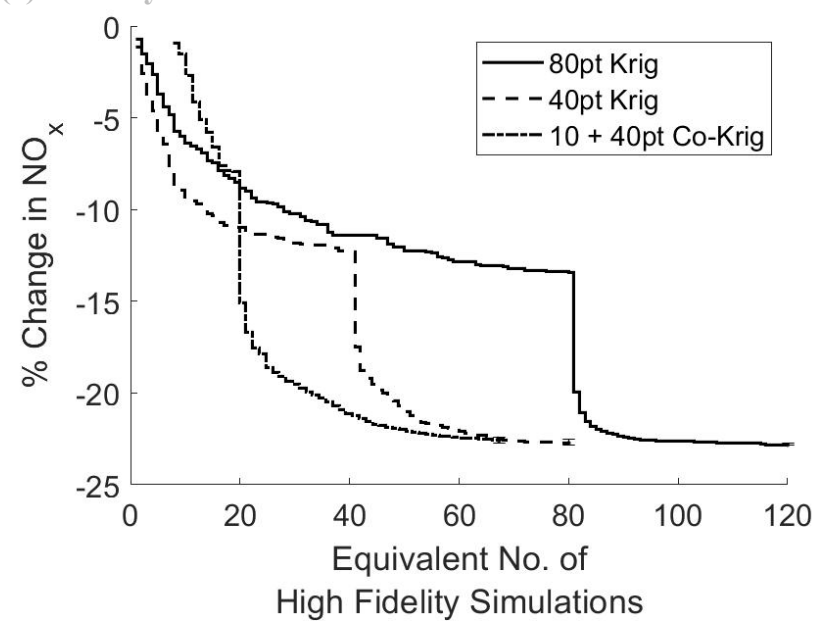

(a)

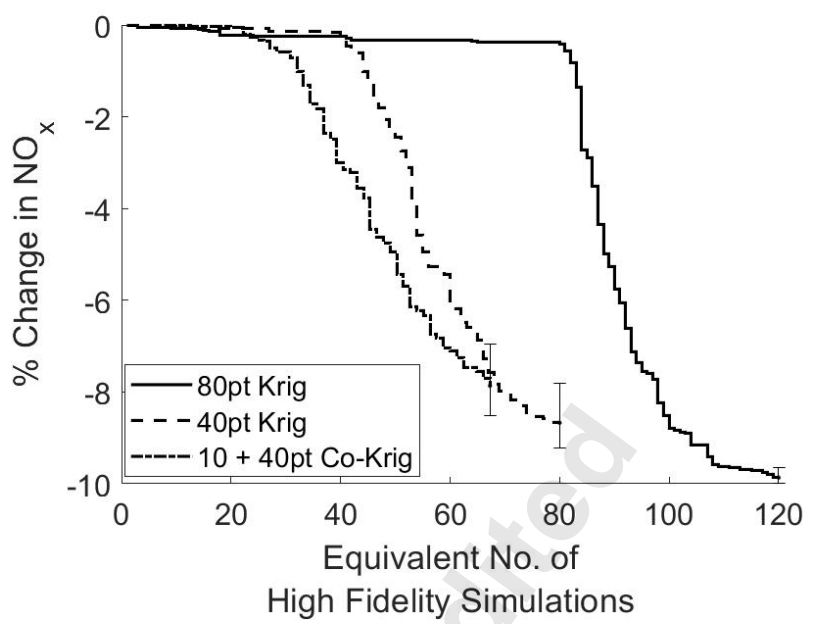

(b)

Fig. 5. Mean search histories for each 8D optimization strategy (a) without soot constraint and (b) with soot constraint active.

single fidelity approach, at least initially. By the end of the optimization it is only marginally ahead of the 40 point single fidelity approach.

\section{16D Case Study}

The final case study extends the dimensionality of the optimization problem to 16 variables. In this instance the axial position and radius of each of the eight individual ports can be controlled independently. Unlike the previous case studies the following optimizations will be performed directly on the CFD simulations instead of evaluations of large surrogate models. While a more realistic test, as it introduces noise and simulation failures into the optimization the cost of carrying out the CFD simulations prohibits averaging of the performance of each strategy over a number of different optimization runs. Each optimization is therefore performed only once.

As with the previous two studies, optimizations with and without the soot constraint is considered along with three different optimization strategies: two single fidelity approaches employing an 80 and 40 point initial sampling plan along with a multi-fidelity approach employing a 20 point initial high fidelity sampling plan combined with a 92 point low fidelity sampling plan. In all cases a total of 20 update cycles are performed. Table 9 describes the relative cost of each strategy and the best design found by each for the two optimisation problems. The multi-fidelity approach, in this instance, is an equivalent cost to the 40 point single fidelity approach.

Table 9. 16 variable optimization strategy cost and improvement in NOx with and without soot constraint included

\begin{tabular}{llll}
\hline \hline Strategy & Cost & $\begin{array}{l}\text { Improvement } \\
\text { (w/o soot) }\end{array}$ & $\begin{array}{l}\text { Improvement } \\
\text { (with soot) }\end{array}$ \\
\hline 80pt Krig & $100 \%$ & $2.94 \%$ & $0.38 \%$ \\
40pt Krig & $60 \%$ & $0.78 \%$ & $4.16 \%$ \\
20+92pt Co-Krig & $60 \%$ & $4.76 \%$ & $5.25 \%$ \\
\hline
\end{tabular}

With or without the soot constraint present the multi-fidelity approach out performs both the 40 and 80 point single fidelity optimizations in terms of the overall improvement in NOx. A result reinforced by the optimization histories presented in Figure 6. Without the soot constraint the multi-fidelity approach converges to a better design considerably faster than the more expensive 80 point optimization which struggles to locate any designs which improve NOx and satisfy the pressure loss constraint in any of the updates after the initial sampling plan. It's only on the penultimate CFD simulation that it achieves any real improvement in performance.

With the soot constraint present the multi-fidelity optimization performs even better. Rather counter-intuitively it is able to locate a design which, despite the presence of the soot constraint, reduces NOx further beyond that obtained without the 
Copyright (c) 2020 bot constraint. 'The 80 point single fidelity approach is unable to locate a design better than that found during it's initial sampling plan despite 20 further CFD simulations in locations of potentially good designs. The 40 point single fidelity optimization, however, actually out performs the more costly 80 point single fidelity optimization in this instance.

Of course, these results are only for a single optimization for each strategy and such optimizations can be heavily dependent on the initial design of experiments sampling plan. This is even more the case when the true function to be optimization is the result of some sort of physics based simulation and therefore potentially subject to considerable levels of noise due to, for example, the discretization scheme, poor convergence, etc. The inclusion of such 'bad' data within the construction of a surrogate model can result in a model which misleads the optimization strategy into exploring the wrong region. The model will, of course, correct itself over time as additional samples are made within this region but this will naturally impact the rate of convergence.

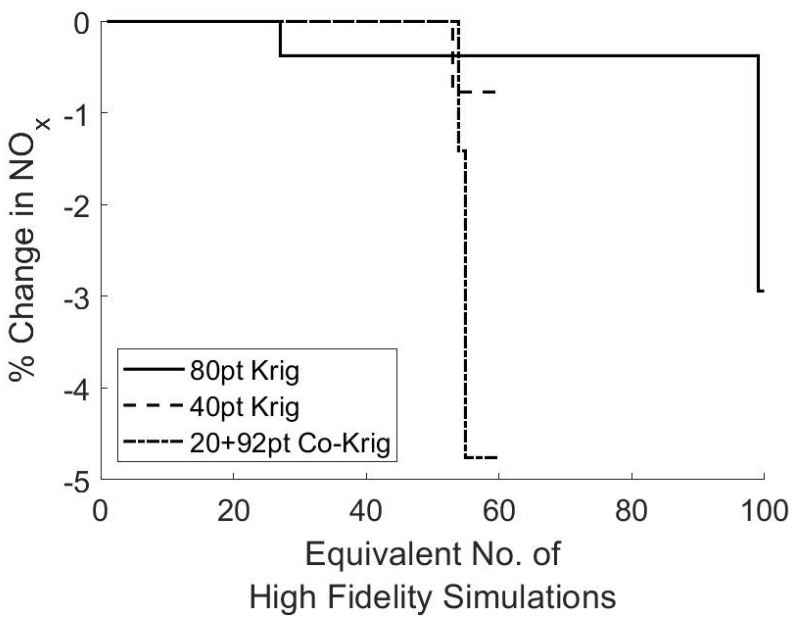

(a)

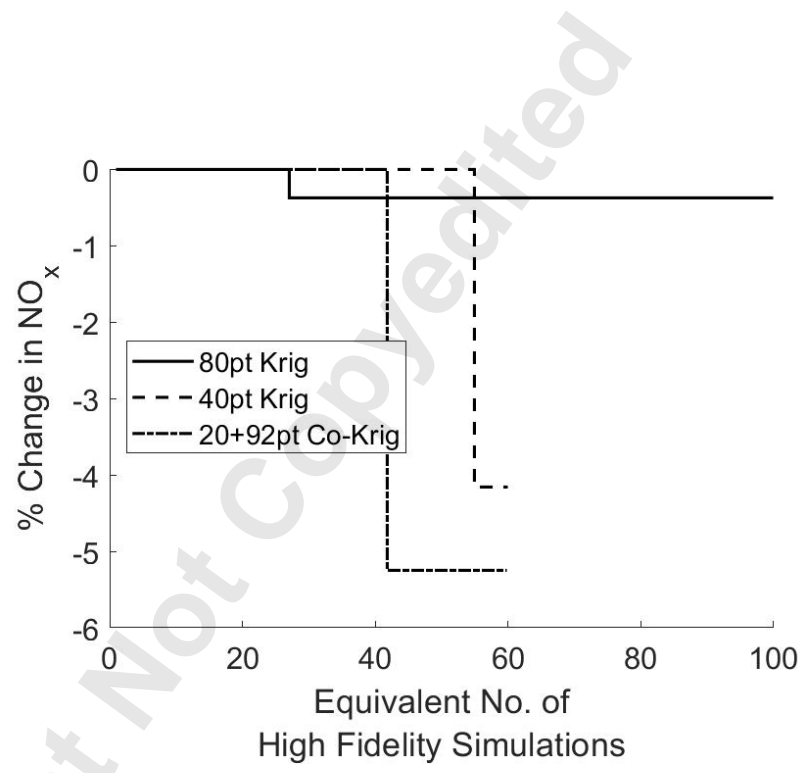

(b)

Fig. 6. Search histories for each 16D optimization strategy (a) without soot constraint and (b) with soot constraint active.

A similar phenomena was encountered by Zhang et al. [16] in their combustor design optimization where an initial poorly converged outlying simulation close to the optimum resulted in a delay in convergence as the model corrected itself. The above results, particularly for the case with the soot constraint included demonstrate the hallmarks of a similar situation occurring where one or more anomalous results present within the larger 80 point sampling plan are actually hampering convergence towards an optimum. By chance these points are not present within the smaller 40 point sampling plan, which is a subset of the 80 point plan.

\section{Conclusions}

The current paper represents the first time that a multi-fidelity optimization approach has been employed in the minimization of combustor emissions. It is the first time that any multi-fidelity combustor optimization has employed a variation in mesh density in order to change the fidelity of a reacting flow simulation. Previously varying levels of geometry fidelity (single vs. double sector) or solution fidelity (RANS vs. URANS CFD or an aerothermal network model) have been used to alter the fidelity. The complexity of the geometry employed within the optimization is also much greater than that typically employed within design studies in the literature and is of a level comparable to that typically employed within industrial design studies.

The results presented above for each of the three case studies demonstrate the potential of a multi-fidelity surrogate modelling based approach applied to the minimization of combustor emissions. In all of the case studies the multi-fidelity approach has either achieved similar or greater reductions in NOx compared to a more expensive single fidelity approach. This holds true whether or not a constraint on the level of soot production is present within the problem definition.

These results indicate that a multi-fidelity approach may offer considerable cost savings in terms of the number of simulations performed as part of a design study. Alternatively a multi-fidelity approach may enable larger studies with greater geometric freedom to be performed in a similar total time thereby potentially unlocking greater emission reductions. 
[1] Forrester, A., Sóbester, A., and Keane, A., 2008. Engineering Design via Surrogate Modelling. Wiley-Blackwell.

[2] Toal, D., 2015. "Some considerations regarding the use of multi-fidelity kriging in the construction of surrogate models". Structural and Multidisciplinary Optimization.

[3] Rogero, J. M., and Rubini, P. A., 2003. "Optimization of combustor wall heat transfer and pollutant emissions for preliminary design using evolutionary techniques". Proceedings of the Institution of Mechanical Engineers, Part A: Journal of Power and Energy, 217(6), sep, pp. 605-614.

[4] Wyse, S. G., Parks, G. T., and Cant, R. S., 2006. "Towards the multiobjective optimisation of gas turbine combustors". In Volume 1: Combustion and Fuels, Education, ASME.

[5] Saboohi, Z., Ommi, F., and Akbari, M., 2019. "Multi-objective optimization approach toward conceptual design of gas turbine combustor". Applied Thermal Engineering, 148, feb, pp. 1210-1223.

[6] Torkzadeh, M., Bolourchifard, F., and Amani, E., 2016. "An investigation of air-swirl design criteria for gas turbine combustors through a multi-objective CFD optimization". Fuel, 186, dec, pp. 734-749.

[7] Deb, K., 2001. Multi-Objective Optimization using Evolutionary Algorithms. Wiley.

[8] Amani, E., Rahdan, P., and Pourvosoughi, S., 2019. "Multi-objective optimizations of air partitioning in a gas turbine combustor". Applied Thermal Engineering, 148, feb, pp. 1292-1302.

[9] Duchaine, F., Morel, T., and Gicquel, M., 2009. "Computational-fluid-dynamics-based kriging optimization tool for aeronautical combustion chambers". AIAA Journal, 47(3), pp. 631-645.

[10] Motsamai, O. S., Snyman, J. A., and Meyer, J. P., 2010. "Optimization of gas turbine combustor mixing for improved exit temperature profile”. Heat Transfer Engineering, 31(5), apr, pp. 402-418.

[11] Laranci, P., Zampilli, M., D’Amico, M., Bartocci, P., Bidini, G., and Fantozzi, F., 2017. "Geometry optimization of a commercial annular RQL combustor of a micro gas turbine for use with natural gas and vegetal oils". Energy Procedia, 126, sep, pp. 875-882.

[12] Briones, A. M., Burrus, D. L., Sykes, J. P., Rankin, B. A., and Caswell, A. W., 2018. “Automated design optimization of a small-scale high-swirl cavity-stabilized combustor". Journal of Engineering for Gas Turbines and Power, 140(12), nov.

[13] Thomas, N., Rumpfkeil, M. P., Briones, A., Erdmann, T. J., and Rankin, B. A., 2019. "Multiple-objective optimization of a subsonic small-scale cavity-stabilized combustor". In AIAA Scitech 2019 Forum, American Institute of Aeronautics and Astronautics.

[14] Wankhede, M., Bressloff, N., and Keane, A., 2011. "Combustor design optimization using co-kriging of steady and unsteady turbulent combustion”. Journal of Engineering for Gas Turbines and Power, 133(12).

[15] Zhang, X., Toal, D., Bressloff, N., Keane, A., Witham, F., Gregory, J., Stow, S., Goddard, C., Zedda, M., and Rodgers, M., 2014. "Prometheus: a geometry-centric optimisation system for combustor design". In ASME Turbo Expo 2014: Turbine Technical Conference and Exposition, Dusseldorf, DE.

[16] Zhang, X., Toal, D. J. J., Keane, A. J., Witham, F., Gregory, J., Ravikanti, M., Aurifeille, E., Stow, S., Rogers, M., and Zedda, M., 2015. "Isothermal combustor prediffuser and fuel injector feed arm design optimization using the prometheus design system". Journal of Engineering for Gas Turbines and Power, 138(6), nov.

[17] Toal, D., Zhang, X., Keane, A., Stow, S., Zedda, M., Witham, F., and Gregory, J., 2017. “Combustor design optimization using the prometheus design system”. In 23rd ISABE Conference, Manchester, United Kingdom.

[18] Jones, D., 2001. “A taxonomy of global optimization methods based on response surfaces". Journal of Global Optimization, 21(4), pp. 345-383.

[19] Forrester, A., Keane, A., and Bressloff, N., 2006. "Design and analysis of "noisy" computer experiments". AIAA Journal, 44(10), pp. 2331-2339.

[20] Toal, D., Bressloff, N., Keane, A., and Holden, C., 2011. "The development of a hybridized particle swarm for kriging hyperparameter tuning". Engineering Optimization (Accepted for Publication).

[21] Toal, D., Forrester, A., Bressloff, N., Keane, A., and Holden, C., 2009. "An adjoint for likelihood maximization". Proceedings of the Royal Society A, 465(2111), pp. 3267-3287.

[22] Kennedy, M., and O'Hagan, A., 2000. "Predicting the output from a complex computer code when fast approximations are available". Biometrika, 87(1), pp. 1-13.

[23] Toal, D., and Keane, A., 2011. "Efficient multi-point aerodynamic design optimization via co-kriging". Journal of Aircraft, 48(5), pp. 1685-1695.

[24] Anand, M. S., Eggels, R., Staufer, M., Zedda, M., and Zhu, J., 2013. "An advanced unstructured-grid finite-volume design system for gas turbine combustion analysis". In ASME 2013 Gas Turbine India Conference, American Society of Mechanical Engineers. 